\title{
RESEARCH ON ONE-DIMENSIONAL UBIQUITIFORMAL CONSTITUTIVE RELATIONS FOR A BIMATERIAL BAR
}

\author{
Min Yang, Zhuo-Cheng Ou, Zhuo-Ping Duan, Feng-Lei Huang \\ State Key Laboratory of Explosion Science and Technology, Beijing Institute of Technology, Beijing, China \\ e-mail: zcou@bit.edu.cn
}

\begin{abstract}
A one-dimensional ubiquitiformal constitutive model for a bimaterial bar is proposed in this paper. An explicit analytical expression for the effective Young modulus is then obtained, which, unlike the fractal one, leads to a continuous displacement distribution along the bar. Moreover, numerical results for concretes are calculated and found to be in agreement with previous experimental data. In addition, some previous empirical and semi-empirical constitutive models are also examined, which shows that each of these models can correspond well to a ubiquitiformal one under a certain complexity.
\end{abstract}

Keywords: ubiquitiform, fractal, composite, displacement distribution, elasticity modulus

\section{Introduction}

Over the past decades, researchers have been concerned with the constitutive relationship of composites, and it is of particular significance to understand how to obtain macroscopic material properties from its microstructure. For this purpose, much theoretical, numerical and experimental work has been carried out, in which the influence of microstructure, properties of individual components of the composites under consideration, as well as the interaction among the components on the macroscopic material behavior are all taken into account. For example, concrete is a one kind of widely used construction materials in practical applications, and several constitutive models for concrete have been proposed, such as the Hirsch model (Hirsch, 1962), the Counto model (Counto, 1964), the Hashin model (Hashin, 1962) and the three-phase composite circle model (Zheng et al., 2006). Moreover, some experimental studies for concrete have also been carried out. For example, Hirsch (1962) investigated experimentally the effects of both the aggregate and the cement paste elastic moduli on the effective secant and dynamic elastic moduli; Stock et al. (1979) performed an experimental research on the effect of the aggregate volume fraction on the strength and the elastic modulus of concrete under both uniaxial tension and compression; Vilardell et al. (1998) obtained experimentally stress-strain curves for mortar, wet-screened concrete and dam concrete at different ages. In addition, numerical simulations for the problem can also be found in the literature. For example, by using the finite element method, Li et al. (2003) predicted the elastic modulus of a kind of three-phase concrete and investigated the influence of the aggregate area fraction and the elastic modulus ratios of both the aggregate and the interfacial transition zone to the cement paste on the overall elastic modulus of concrete; Häfner et al. (2006) used fast separation checks to place ellipsoidal and arbitrary shaped particles and studied the influence of the concrete mix parameters and the particle shape on the effective elastic properties.

On the other hand, since the pioneer work of Mandelbrot $(1967,1977,1982)$ and Mandelbrot et al. (1984), fractals have been extensively used to describe constitutive relations of composites. 
For example, based on the concepts of fractal strain and fractal stress, Carpinteri and Cornetti (2002) obtained the constant displacement and the constant elastic modulus of a one-dimensional stretched fractal bar under a uniaxial tensile load by using the renormalization group procedure to the fractal Cantor set. Recently, Davey and Alonso Rasgado (2011) developed a new fractal analytical procedure to obtain the finite displacement and the finite strain energy for a onedimensional bi-material bar. By constructing a map between a pre-fractal Cantor bar and a continuous one-dimensional bar, they obtained first an analytical solution on the pre-fractal bar from the solution of the effective continuum model, and then an analytical expression for the fractal bi-material bar was obtained by an infinite limit process of the solution of the pre-fractal one. However, there are still some perplexities in the two fractal models (Carpinteri and Cornetti, 2002; Davey and Alonso Rasgado, 2011). First, to determine the finite displacement, elastic modulus and strain energy, the new density kinds of fractal parameters such as the fractal strain, the fractal stress and the fractal length that defined on a unit fractal measure would be used. However, as have been recently pointed out by Ou et al. (2014), such a concept of fractal parameters seems now a little questionable, because that these fractal parameters are both difficult to be determined in practice and have unambiguous physical meanings (Bažant and Yavari, 2005). Next, the corresponding displacement distribution is in the form of a likely devil's staircase (see Fig. 1 and Fig. 4 in Carpinteri and Cornetti (2002), and Fig. 2 in Davey and Alonso Rasgado (2011)), which is not continuous in internal boundaries between the two components of the bi-material Cantor bar, and this is not the case for a real material in nature. Finally, theoretically, such a solution can only be adopted for an idealized composite material consisting of rigid aggregates and matrix layers of vanishing length and the elastic modulus, which is apparent inconformity with the actual materials. In fact, in the opinions of the authors of this paper, the aforementioned perplexities are resulted directly from the infinite iterative procedure of fractals. According to Ou et al. (2014), the fractal approximation of a real physical or geometrical object is questionable, especially when the measure of the considered object must be taken into account, simply because of the divergence of the integral dimensional measure or the singularity of the Hausdorff dimension of a fractal. In order to avoid such a problem in fractal applications, a new concept of ubiquitiform was introduced by $\mathrm{Ou}$ et al. (2014), according to which, a ubiquitiform is a finite order self-similar (or self-affine) physical configuration constructed usually by a finite iterative procedure. In particular, a ubiquitiform has the same Hausdorff dimension as that of the initial element, which is always integral in practice, and a physical object in nature is a ubiquitiform. Recently, ubiquitiform has been applied successfully in the softening constitutive model of concrete (Ou et al., 2019), heat transfer in a bimaterial bar ( $\mathrm{Li}$ et al., 2016), crack extension in concrete (Li et al., 2017), and fracture energy of concrete (Ou et al., 2017).

In this paper, based on the concept of ubiquitiform, a ubiquitiformal constitutive model is proposed to describe a one-dimensional bi-material bar under uniaxial tension, in which a generalized ubiquitiformal ternary Cantor set is used to describe the distribution of the matrix material, and then explicit analytical expressions for both the effective elastic modulus and the displacement distribution are obtained. Moreover, based on the ubiquitiformal constitutive model, the effective elastic moduli for some concrete materials are calculated numerically, and the calculated results are compared with the previous experimental data. This article is divided into the following five sections. After this brief introduction, in Section 2, a one-dimensional ubiquitiformal elastic constitutive model for a bi-material bar is developed, also presented are the explicit analytical expression for both the displacement distribution and the effective elastic modulus. In Section 3, the numerical results are presented and compared with the previous experimental data, and some discussions are presented in Section 4. Finally, in Section 5, some conclusions are given. 


\section{Ubiquitiformal constitutive model for a bi-material bar}

According to $\mathrm{Ou}$ et al. (2014), as a ubiquitiform, a physical object displays self-similarity only in a finite scale range $\left[\delta_{\min }, \delta_{\max }\right]$, where $\delta_{\min }$ and $\delta_{\max }$ are the lower and upper bound to scale invariance, respectively. For a ubiquitiformal elementary cell of length $\delta_{\max }$ in a bimaterial bar, the distributions of the matrix and the aggregate can be described properly by an $N$-th order generalized ubiquitiformal ternary Cantor set that is constructed by a series of deletion process. That is, the initial line element $I_{0}=\left[0, \delta_{\max }\right]$ is first divided into $p$ equal parts, then the middle $p-2$ parts are deleted; subsequently, each remaining part is again divided into $p$ equal smaller parts, and the middle $p-2$ of the smaller parts are also deleted, and so on. After $N$ times of such a deletion operation, an $N$-th order generalized ubiquitiformal ternary Cantor set is generated, as shown in Fig. 1.

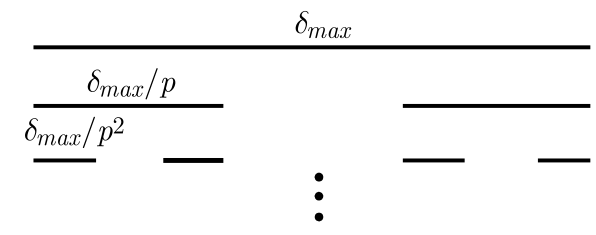

Fig. 1 . The $N$-th order generalized ubiquitiformal ternary Cantor set

In fact, this generalized ubiquitiformal ternary Cantor set can also be obtained from the initial line element through a series of repeated self-similar compression maps in the following form

$$
S:\left\{\begin{array}{l}
S_{1}(x)=\frac{x}{p} \\
S_{2}(x)=\frac{x+(p-1) \delta_{\max }}{p}
\end{array}\right.
$$

by which, the $N$-th order generalized ubiquitiformal ternary Cantor set can be obtained after $N$ times of iteration, as

$$
I_{N}=S^{N}\left(I_{0}\right)=\bigcup_{j=1}^{2} S_{j}\left(I_{N-1}\right)
$$

and the complexity of the $N$-th order generalized ubiquitiformal ternary Cantor set is

$$
D=\frac{\ln 2}{\ln p}
$$

Physically, as is shown in Fig. 2, in the ubiquitiformal elementary cell, elements I (remaining parts) are assumed to consist of only the matrix material, and elements II (removed parts) are assumed to include the aggregate and some of the matrix material.

Moreover, according to Li et al.(2016), there is

$$
\left(\frac{1}{p}\right)^{N}=\frac{\delta_{\min }}{\delta_{\max }}
$$

Eliminating the variable $p$ from Eq. (2.3) and Eq. (2.4) gives

$$
N=-D \frac{\ln \left(\delta_{\min } / \delta_{\max }\right)}{\ln 2}
$$




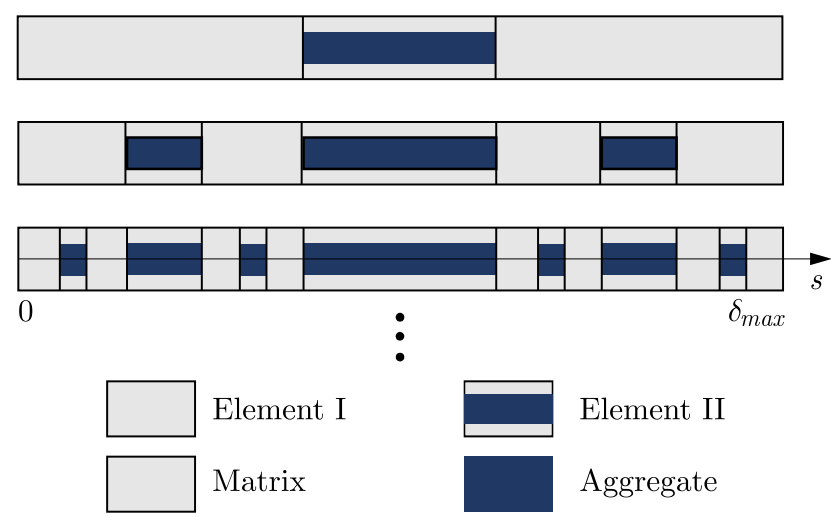

Fig. 2. The distribution of the matrix material and aggregate in the bi-material bar

For convenience, from Eq. (2.4) and Eq. (2.5), the length ratio of the generalized Cantor set $(2 / p)^{N}$ can be written as

$$
\left(\frac{2}{P}\right)^{N}=\left(\frac{\delta_{\min }}{\delta_{\max }}\right)^{(1-D)}
$$

Denoting the elastic modulus of the matrix material, the aggregate, elements I and elements II as $E_{m}, E_{a}, E_{\mathrm{I}}\left(=E_{m}\right)$ and $E_{\mathrm{II}}$, respectively, and the cross-sectional area of the bar and of the aggregate and matrix in element II as $A, A_{a}$ and $A_{m}=A-A_{a}$, respectively, it can be easily shown that the effective elastic modulus of element II $E_{\mathrm{II}}$ is

$$
E_{\mathrm{II}}=E_{m}+\left(E_{a}-E_{m}\right) \frac{A_{a}}{A}
$$

To determine $A_{a}$, the following expression is used

$$
V_{a}=\left[1-\left(\frac{\delta_{\min }}{\delta_{\max }}\right)^{(1-D)}\right] \delta_{\max } A_{a}=r_{a} \delta_{\max } A
$$

where $V_{a}$ is the volume of the aggregate included in the bimaterial bar, and $r_{a}$ is the ratio of the aggregate volume to the total volume. Substituting Eq. (2.8) into Eq. (2.7), reaches

$$
E_{\mathrm{II}}=E_{m}+\frac{r_{a}\left(E_{a}-E_{m}\right)}{1-\left(\delta_{\min } / \delta_{\max }\right)^{(1-D)}}
$$

To determine the effective elastic modulus $E_{\text {eff }}$ of the bimaterial bar, the displacement distribution along the ubiquitiformal elementary cell under a certain external load must be first determined, which will be described in the following.

Consider the displacement distribution of a ubiquitiformal elementary cell fixed at the left end under an axial external load $F=\sigma A$, where $\sigma$ is the stress acting on the cross section. The displacement at the location $s$ along the ubiquitiformal elementary cell is $u^{N}(s)=\Delta u^{N}(s)$, where $\Delta u^{N}(s)$ is the extension of the line segment $[0, s]$. Denoting the total initial lengths of elements I and II in the line segment $[0, s]$ as $\Delta_{\mathrm{I}}(s)$ and $\Delta_{\mathrm{II}}(s)=s-\Delta_{\mathrm{I}}(s)$, respectively, $u^{N}(s)$ can be expressed by

$$
u^{N}(s)=\Delta_{\mathrm{I}}(s) \frac{F}{E_{\mathrm{I}} A}+\left[s-\Delta_{\mathrm{I}}(s)\right] \frac{F}{E_{\mathrm{II}} A}
$$

Moreover, it is known that there are totally $2^{N}$ elements I in the $N$-th ubiquitiformal elementary cell. Denoting the coordinates of the left and the right end of the $j$-th element I as $x_{j}^{N}$ 
$\left(j=1, \ldots, 2^{N}\right)$ and $y_{j}^{N}\left(j=1, \ldots, 2^{N}\right)$, respectively, with the boundary conditions of $x_{1}^{N}=0$ and $y_{2^{N}}^{N}=\delta_{\max }$, both $x_{j}^{N}$ and $y_{j}^{N}$ could be obtained by the self-similar compression mapping Eq. (2.1). Therefore, for $s \in\left(y_{j}^{N}, x_{j+1}^{N}\right), j=1, \ldots, 2^{N-1}$, namely, the point $s$ belonging to the $j$-th element II, there is $\Delta_{\mathrm{I}}(s)=j(1 / p)^{N} \delta_{\max }$; for $s \in\left(x_{j}^{N}, y_{j}^{N}\right), j=1, \ldots, 2^{N}$, namely, the point $s$ belonging to the $j$-th element $\mathrm{I}$, there is $\Delta_{\mathrm{I}}(s)=(j-1)(1 / p)^{N} \delta_{\max }+s-x_{j}^{N}$. Accordingly, taking Eq. (2.4) into account, one obtains

$$
\Delta_{\mathrm{I}}(s)= \begin{cases}j \delta_{\text {min }} & y_{j}^{N} \leqslant s<x_{j+1}^{N} \\ (j-1) \delta_{\text {min }}+s-x_{j}^{N} & x_{j}^{N} \leqslant s<y_{j}^{N}\end{cases}
$$

For the sake of convenience, $\Delta_{\mathrm{I}}(s)$ can be denoted as

$$
\Delta_{\mathrm{I}}(s)=\int_{0}^{s} \mu_{\mathrm{I}}(s) d s
$$

where

$$
\mu_{\mathrm{I}}(s)= \begin{cases}1 & s \in \text { element I } \\ 0 & s \in \text { element II }\end{cases}
$$

Substituting Eqs. (2.9) and (2.12) into Eq. (2.10), one gets the displacement distribution along the bimaterial bar

$$
u^{N}(s)=\frac{F}{E_{m} A}\left[\int_{0}^{s} \mu_{\mathrm{I}}(\eta) d \eta+\frac{s-\int_{0}^{s} \mu_{\mathrm{I}}(\eta) d \eta}{1+\left(\frac{E_{a}}{E_{m}}-1\right) \frac{r_{a}}{1-\left(\delta_{\min } / \delta_{\max }\right)^{(1-D)}}}\right]
$$

and the total extension of the $N$-th order ubiquitiformal elementary cell $\Delta u^{N}$ is

$$
\Delta u^{N}=\frac{F}{E_{m} A} \delta_{\max }\left[\left(\frac{\delta_{\min }}{\delta_{\max }}\right)^{(1-D)}+\frac{1-\left(\delta_{\min } / \delta_{\max }\right)^{(1-D)}}{1+\left(\frac{E_{a}}{E_{m}}-1\right) \frac{r_{a}}{1-\left(\delta_{\min } / \delta_{\max }\right)^{(1-D)}}}\right]
$$

On the other hand, by the linear elastic constitutive relation, the total extension can be expressed as

$$
\Delta u^{N}=\frac{F}{A E_{\text {eff }}} \delta_{\max }
$$

hence, from Eqs. $(2,15)$ and $(2.16)$, one obtains the effective elastic modulus of the bimaterial bar

$$
E_{\text {eff }}=\frac{E_{m}}{\left(\frac{\delta_{\min }}{\delta_{\max }}\right)^{(1-D)}+\frac{1-\left(\delta_{\min } / \delta_{\max }\right)^{(1-D)}}{1+\left(\frac{E_{a}}{E_{m}}-1\right) \frac{r_{a}}{1-\left(\delta_{\min } / \delta_{\max }\right)^{(1-D)}}}}
$$

\section{Numerical results and discussions}

Substituting Eq. (2.6) into Eq. (2.14), yields

$$
u^{N}(s)=\frac{F}{E_{m} A}\left[\int_{0}^{s} \mu_{\mathrm{I}}(\eta) d \eta+\frac{s-\int_{0}^{s} \mu_{\mathrm{I}}(\eta) d \eta}{1+\left(\frac{E_{a}}{E_{m}}-1\right) \frac{r_{a}}{1-(2 / p)^{N}}}\right]
$$


To obtain the displacement distribution in the $N$-th order basic ubiquitiformal cell of a bimaterial bar under uniaxial tension by using Eq. (3.1), some necessary material parameters must be determined first, which include the lower and the upper bound to scale invariance $\delta_{\min }$ and $\delta_{\max }$, respectively, the ratio $r_{a}$ of the aggregate volume to the total volume, and the elastic moduli of the matrix material $E_{m}$ and the aggregate $E_{a}$. Moreover, the complexity $D$ is obtained directly by the mass distribution function or the gradation and the volume ratio of the aggregate (Carpinteri et al., 2003), and then the parameter $p$ and $N$ can be determined from Eq. (2.3) and Eq. (2.4), respectively.

For a one kind of practical concrete materials (Stock et al., 1979), there are $E_{m}=11.6 \mathrm{GPa}$, $E_{a}=74.5 \mathrm{GPa}$, and $r_{a}=60 \%$. According to Ou et al. (2019), for concrete, the lower bound to scale invariance $\delta_{\min }$ can be expressed as a function of the tensile strength, as

$$
\delta_{\text {min }}=221.38 f_{t}^{-3.24}
$$

where $f_{t}$ is the tensile strength of the concrete, and the unit of $\delta_{\min }$ and $f_{t}$ are $\mu \mathrm{m}$ and $\mathrm{MPa}$, respectively. For the concrete presented in Stock et al. (1979), $f_{t}=2.38 \mathrm{MPa}$, and then $\delta_{\text {min }}=13.33 \mu \mathrm{m}$. Moreover, by using gradation curve 3 of the aggregate in Road Note 4 (Road Research laboratory, 1950), the mass distribution function of the aggregate can be fitted as $W(d)=\left(d / d_{\max }\right)^{0.68}$, where $d$ is the diameter of the aggregate, and the maximum diameter of the aggregate $d_{\max }=19 \mathrm{~mm}$. For a representative volume, the upper bound to scale invariance is taken as $\delta_{\max }=5 d_{\max }=95 \mathrm{~mm}$. Furthermore, from the mass distribution function of the aggregate $W(d)$, the probability density function for the size distribution of the aggregate can be obtained as $f(d)=2.32 d_{\text {min }}^{2.32} / d^{3.32}$, where $d_{\text {min }}=150 \mu \mathrm{m}$. Subsequently, by a numerical integral approach (Carpinteri et al., 2003), the complexity of any cross section of the bimaterial bar can be determined as 1.86, and then the complexity of the generalized ubiquitiformal Cantor set can be determined approximately as $D=0.86$. Finally, substituting the value of the complexity $D$ into Eq. (2.3) gives $p=2.24$, and then, from Eq. (2.5), $N=12$ can be determined.

By virtue of the above obtained values of the material parameters, the numerical result of the dimensionless displacement distribution along the $N$-th order ubiquitiformal elementary cell can be calculated from Eq. (3.1) and presented in Fig. 3.

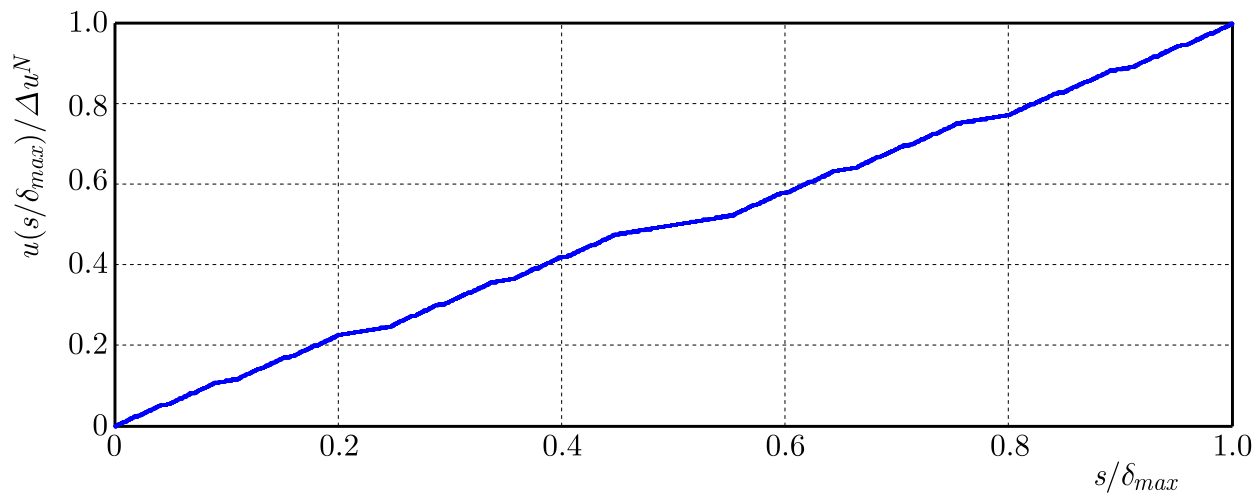

Fig. 3. Dimensionless displacement distribution along the $N$-th ubiquitiformal elementary cell for a one kind of concrete (Stock et al., 1979)

It is interesting and of great significance to compare this numerical result with that obtained from some fractal models (Carpinteri and Cornetti, 2002; Davey and Alonso Rasgado, 2011). It can be seen from Fig. 3 that there is an approximately linear distribution of the particle displacement along the ubiquitiformal elementary cell, which implies also a linear constitutive relation for the bimaterial bar under the tensile loads. Moreover, it can be noticed from Fig. 3 that the displacement distribution is continuous even at any internal interface between the two materials, which is consistent with the experimental observations under the uniaxial tension. 
On the contrary, however, by using the fractal models for the same problem, the corresponding displacement distribution forms a likely devil's staircase as shown in Fig. 1 and Fig. 4 in Carpinteri and Cornetti (2002), and Fig. 2 in Davey and Alonso Rasgado (2011), which is obviously not the case, although it was declared (Carpinteri and Cornetti, 2002; Davey and Alonso Rasgado, 2011) that the fractal model can be applied to an idealized composite material consisting of relatively small particles of infinitely high elasticity modulus embedded in a relatively large matrix of an infinitely low elasticity modulus. In fact, from the physical point of view, such an idealized fractal composite material implies that $E_{1}=0$ and $E_{2}=\infty$, which is not reasonable. Theoretically, such a difficulty comes from the infinite iterative procedure of a fractal set, or, in other words, from the singularity of the integer dimensional measure of a fractal set. Moreover, from Eq. (2.17), the numerical results of the effective elastic moduli for the four kinds of concrete with different aggregate volume ratio (Stock et al., 1979) are obtained, as listed in Table 1. Also listed, for comparison, are the material properties and parameters of the four kinds of concrete, where $E_{\text {exp }}$ represents the experimental data of the effective elastic modulus, and $e_{r}$ is the relative error of $E_{\text {eff }}$ with respect to $E_{\text {exp }}$. It can be seen from Table 1 that the calculated numerical results are basically in agreement with the experimental results.

Table 1. The material properties and the comparison between the numerical results of the effective elastic modulus of concrete and the experimental data (Stock et al., 1979)

\begin{tabular}{|c|c|c|c|c|c|c|c|c|c|}
\hline $\begin{array}{c}r_{a} \\
{[\%]}\end{array}$ & $\begin{array}{c}f_{t} \\
{[\mathrm{MPa}]}\end{array}$ & $\begin{array}{c}\delta_{\min } \\
{[\mu \mathrm{m}]}\end{array}$ & $\begin{array}{c}\delta_{\max } \\
{[\mathrm{mm}]}\end{array}$ & $\begin{array}{c}E_{m} \\
{[\mathrm{GPa}]}\end{array}$ & $\begin{array}{c}E_{a} \\
{[\mathrm{GPa}]}\end{array}$ & $\begin{array}{c}D \\
{[-]}\end{array}$ & $\begin{array}{c}E_{\text {eff }} \\
{[\mathrm{GPa}]}\end{array}$ & $\begin{array}{c}E_{\text {exp }} \\
{[\mathrm{GPa}]}\end{array}$ & $\begin{array}{c}e_{r} \\
{[\%]}\end{array}$ \\
\hline \hline 20 & 1.84 & 30.69 & 95 & 11.6 & 74.5 & 0.97 & 14.11 & 17.8 & -20.73 \\
\hline 40 & 1.86 & 29.63 & 95 & 11.6 & 74.5 & 0.93 & 17.91 & 21.4 & -16.31 \\
\hline 60 & 2.38 & 13.33 & 95 & 11.6 & 74.5 & 0.86 & 27.43 & 29.0 & -5.41 \\
\hline 80 & 2.78 & 8.06 & 95 & 11.6 & 74.5 & 0.72 & 48.58 & 41.3 & +17.63 \\
\hline
\end{tabular}

It is also seen from Eq. (2.17) that the effective elastic modulus $E_{\text {eff }}$ of a bimaterial bar depends on the aggregate volume ratio $r_{a}$ and the complexity $D$ as well as on the lower and the upper bound to scale invariance. In the following, it will further be shown that, under certain $\delta_{\min }, \delta_{\max }$ and $r_{a}$, the variation of the effective elastic modulus for the bimaterial bar will be limited to a finite range of $\left[E_{\text {eff min }}, E_{\text {eff } \max }\right]$. In the physical point of view, the infimum $E_{\text {eff min }}$ and supremum $E_{\text {eff max }}$ represent the effective elastic modulus of the bimaterial bar under the series and the parallel modes, respectively, of the distribution of the matrix material and the aggregate. In fact, it can be solved from Eq. (2.8) that

$$
D=1-\frac{\ln \left(1-r_{a} A / A_{a}\right)}{\ln \left(\delta_{\min } / \delta_{\max }\right)}
$$

Thus, under certain $\delta_{\min }, \delta_{\max }$ and $r_{a}$, it can be verified from Eq. (3.3) that the complexity $D$ is a monotone increasing function of $A_{a} / A$, and reaches up to its maximum at $A_{a} / A=1$, at which the distribution of the matrix material and aggregate takes the series mode. Accordingly, there is

$$
D_{\max }=1-\frac{\ln \left(1-r_{a}\right)}{\ln \left(\delta_{\min } / \delta_{\max }\right)}
$$

On the other hand, considering the mass conservation of the aggregate under a certain value of $r_{a}$, the parallel mode of the distribution of the matrix material and aggreate can be realized when the total dirtribution length of the aggregate $l_{a}$ reaches up to its maximum, namely, the length of a ubiquitiformal elementary cell $\delta_{\max }$, which implies that $p \rightarrow \infty$, and then, from Eq. (2.3), there is

$$
D_{\min }=0
$$




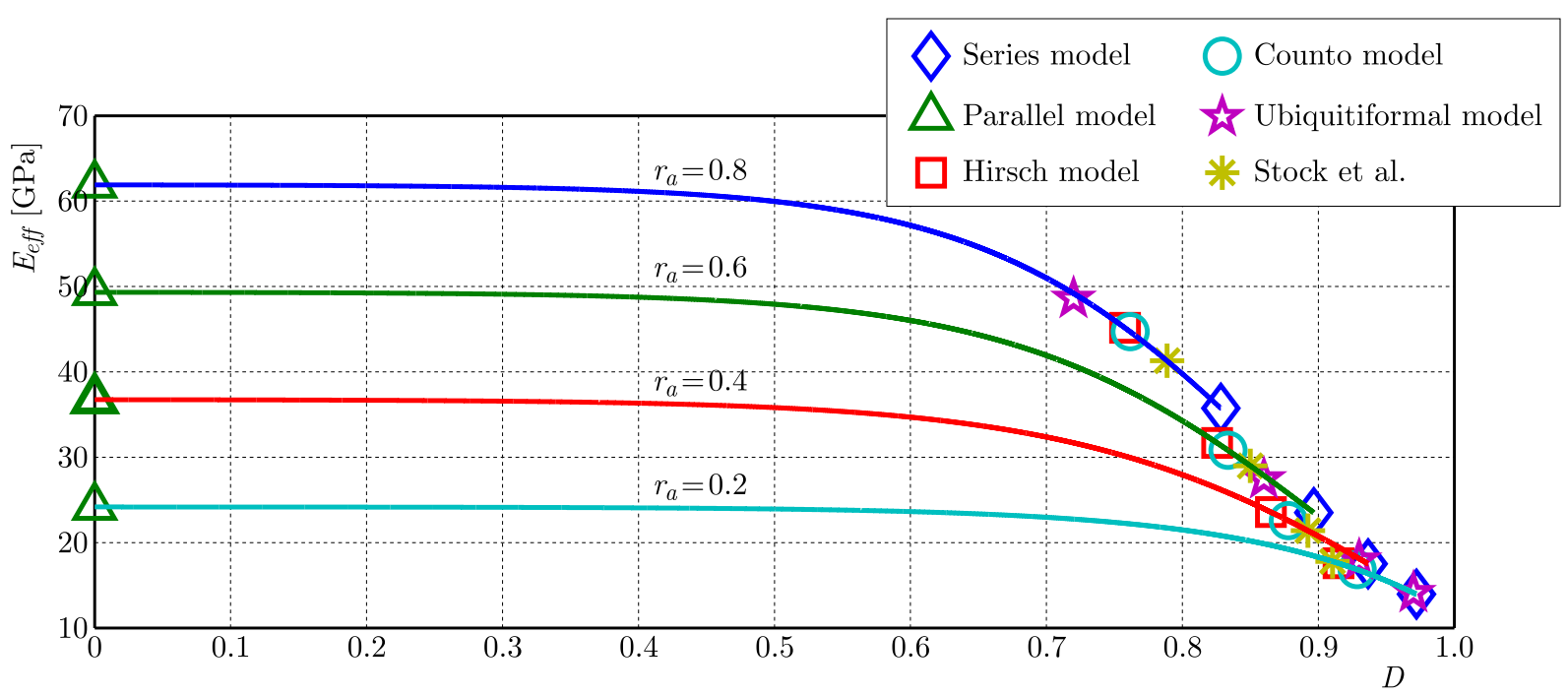

Fig. 4. The relationship between $E_{\text {eff }}$ and $D$ for the concretes (Stock et al., 1979)

Finally, the numerical results of the relationship between the effective elastic modulus $E_{\text {eff }}$ and the complexity $D$ for concretes with different aggregate volume ratio $r_{a}$ (Stock et al., 1979) are calculated and presented in Fig. 4, and that for the relationship between the effective elastic modulus $E_{\text {eff }}$ and the aggregate volume ratio $r_{a}$ under different values of the complexity $D$ are also calculated and presented in Fig. 5. Moreover, the calculated numerical results of the function $E_{\text {eff }}\left(D, r_{a}\right)$ can be presented as a curve surface in the three dimensional space as shown in Fig. 6.

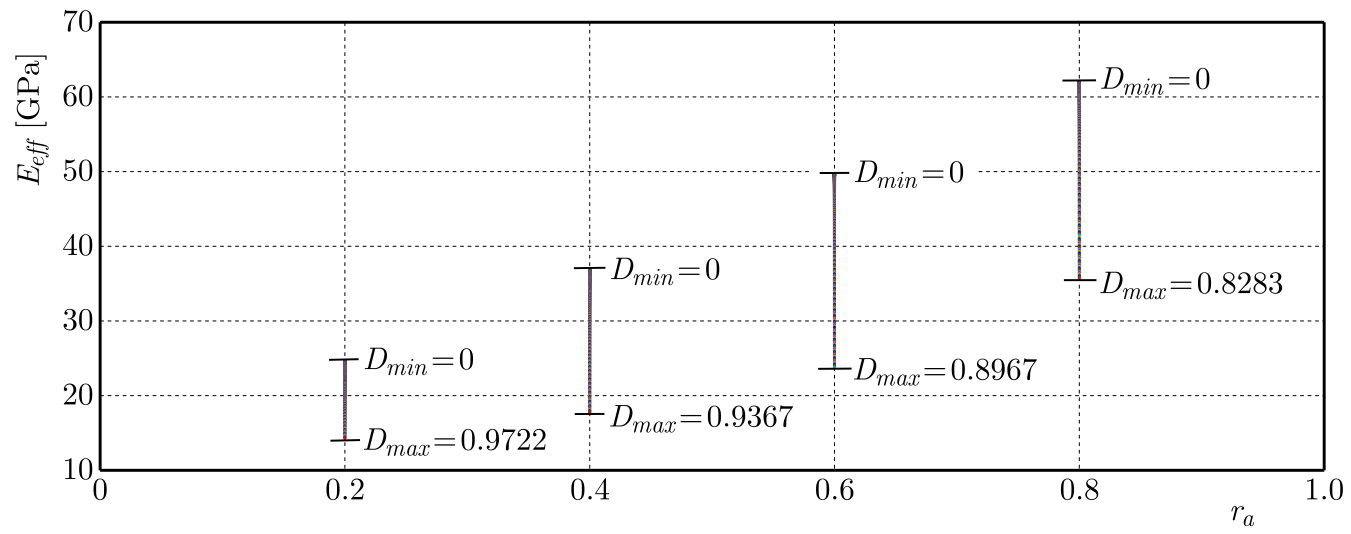

Fig. 5. The relationship between $E_{\text {eff }}$ and $r_{a}$ for the concretes (Stock et al., 1979)

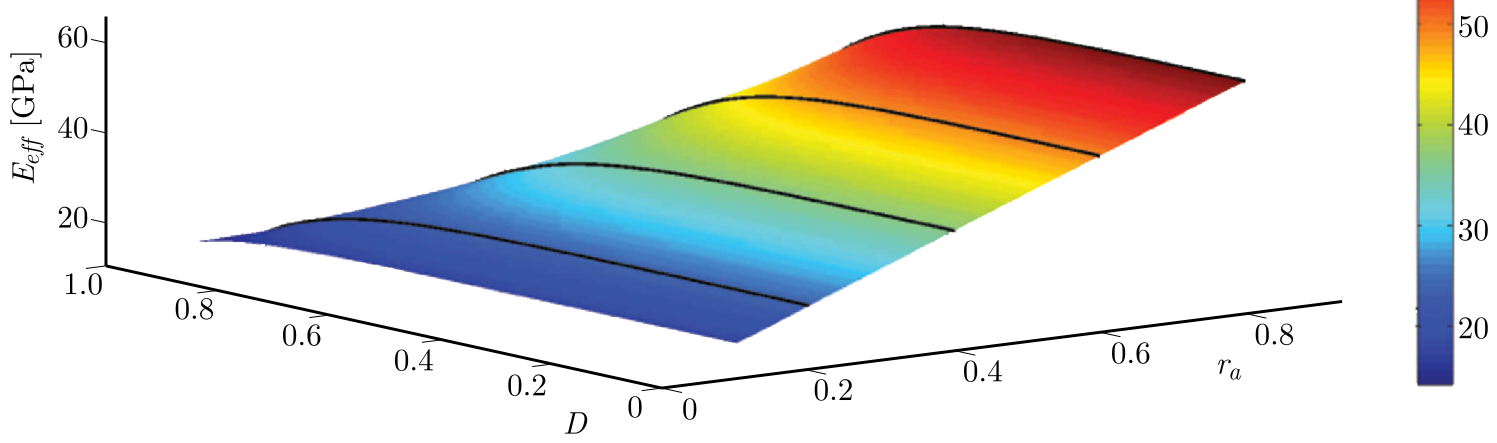

Fig. 6. The dependence of $E_{\text {eff }}$ on $r_{a}$ and $D$ for the concretes (Stock et al., 1979) 


\section{Discussions}

It is seen from the above descriptions that the measures of all the physical quantities used in the ubiquitiformal model are regular on the integer dimensions and can be measured normally. The ubiquitiformal displacement distribution is also continuous, in line with the continuity assumption of traditional continuum mechanics, which implies the availability of the ubiquitiformal model. On the other hand, in the fractal description of such a problem, some embarrassing questions are inevitable, because of the singularity of the integral dimensional measure of the object under consideration and the unreasonable fractal approximation to a ubiquitiform, as have pointed out by Ou et al. (2014). For example, according to Carpinteri and Cornetti (2002), to maintain a finite displacement, all the measures of some physical quantities such as the strain, stress and the cross-sectional area become singular, or, in other words, the concept of the so-called fractal strain, the fractal stress and the fractal area must be introduced. Consequently, a discontinuous displacement distribution is inevitable, which obviously violates the fundamental assumption of continuity in traditional continuum mechanics. Similarly, Davey and Alonso Rssgado (2011) proposed a piecewise-linear mapping between a one-dimensional rod and an $n$ order pre-fractal ternary Cantor set, by which the fractal solution is obtained by taking the limit $n \rightarrow \infty$. However, during the infinite limiting process, the dimensions of some physical quantities such as the stress and the Young modulus change abruptly, which implies that some fractal physical quantities must be introduced. It should be emphasized here that waking up to the questions resulted from the singularity of the integral dimensional measure in practical applications is of important significance. On the one hand, the measure is related to the fractal dimension, so it is meaningless to compare the measures of two objects with different fractal dimensions, but most of the scientific criteria are established based on such a comparison. For example, it is obviously meaningless to compare the values of the fracture energy of two fracture surfaces with different fractal dimensions. On the other hand, the fractal quantities such as the fractal strain, fractal stress, fractal strength and the fractal fracture energy defined on the unit fractal measure are both difficult to be determined in practice and have a unambiguous physical meaning. However, these difficulties will be avoided completely with the concept of a ubiquitiform.

Certainly, there have been some constitutive relations for a two-phase material, and some models are proposed to determine the Young modulus. For instance, besides the above mentioned series and parallel models, there are the Hirsch (1962) and the Counto (1964) models which can be written, respectively, as

$$
\frac{1}{E_{\text {eff }}}=\left(1-\frac{2 Z}{\pi}\right)\left(\frac{r_{a}}{E_{a}}+\frac{1-r_{a}}{E_{m}}\right)+\frac{2 Z}{\pi}\left(\frac{1}{r_{a} E_{a}+\left(1-r_{a}\right) E_{m}}\right) \quad Z=0.785
$$

and

$$
\frac{1}{E_{\text {eff }}}=\frac{1-\sqrt{r_{a}}}{E_{m}}+\frac{1}{E_{m}\left(1-\sqrt{r_{a}}\right) / \sqrt{r_{a}}+E_{a}}
$$

In principle, each of these models is constructed under certain empirical assumptions of the geometric direction of the matrix material and aggregate, with some empirical parameters such as $Z$ in the Hirsch model introduced. Comparing with these existing empirical or semi-empirical models, the ubiquitiformal model confirms the ubiquitiformal characteristic of the distribution of the matrix material and aggregate, which leads to a more definite description of the internal structure of bimaterials. Moreover, the ubiquitiformal model can describe the constitutive behavior of a bimaterial in a more all-round way. For example, besides the aggregate volume ratio $r_{a}$, the aggregate gradation, another important material property, can also be taken into account in the ubiquitiformal model. For comparison, the numerical results of the effective Young modulus 
for the concretes (Stock et al., 1979) calculated by using the ubiquitiformal model and the four above-mentioned models are presented in Fig. 4, in which the complexities of the series, parallel, the Hirsch and the Counto models are all calculated from Eq. (2.17). It can be seen that, in fact, all the four aforementioned models correspond to a ubiquitiformal model under a certain complexity.

In addition, from Eq. (2.17), the numerical results of the effective elastic modulus for the four kinds of concrete with different aggregate volume ratio (Stock et al., 1979) are obtained and listed in Table 2. $E_{\text {eff } 1}$ represents the effective elastic modulus when the complexity is $D_{1}$, and $e_{r}(D)$ and $e_{r}(E)$ are the relative errors of $D_{1}$ with respect to $D$ and the relative errors of $E_{\text {eff } 1}$ with respect to $E_{\text {eff }}$, respectively. It can be seen from Table 2 that the complexity $D$ strongly influences the value of the effective elastic modulus $E_{\text {eff }}$. Moreover, it can also be seen that much attention should be paid in the future to determination of the complexity $D$ in order to further improve the numerical results.

Table 2. The influence of the complexity $D$ on the effective elastic modulus $E_{\text {eff }}$ of the concrete (Stock et al., 1979)

\begin{tabular}{|c|c|c|c|c|c|c|}
\hline $\begin{array}{c}r_{a} \\
{[\%]}\end{array}$ & $\begin{array}{c}D \\
{[-]}\end{array}$ & $\begin{array}{c}E_{\text {eff }} \\
{[\mathrm{GPa}]}\end{array}$ & $\begin{array}{c}D_{1} \\
{[-]}\end{array}$ & $\begin{array}{c}E_{\text {eff } 1} \\
{[\mathrm{GPa}]}\end{array}$ & $\begin{array}{c}e_{r}(D) \\
{[\%]}\end{array}$ & $\begin{array}{c}e_{r}(E) \\
{[\%]}\end{array}$ \\
\hline \hline 20 & 0.97 & 14.11 & 0.96 & 14.86 & -1.03 & +5.31 \\
\hline 40 & 0.93 & 17.91 & 0.92 & 19.03 & -0.01 & +6.21 \\
\hline 60 & 0.86 & 27.43 & 0.85 & 29.00 & -1.16 & +5.73 \\
\hline 80 & 0.72 & 48.58 & 0.71 & 50.11 & -1.39 & +3.16 \\
\hline
\end{tabular}

The evaluation of the effective elastic modulus is applicable for tension. Certainly, further discussions on torsion and bending are still needed in future.

\section{Conclusion}

A ubiquitiformal linear elastic constitutive model for a bimaterial bar is proposed, in which, the bimaterial bar is regarded as a generalized ubiquitiformal ternary Cantor set. Based on this new ubiquitiformal model, the explicit analytical expressions for the effective Young modulus and the displacement distribution are obtained. The ubiquitiformal model leads to a reasonably continuous displacement distribution along the bimaterial bar, while the corresponding fractal displacement distribution is a likely devil's staircase.

Moreover, taking the aggregate volume ratio and the aggregate gradation into account, the numerical results of the effective Young modulus for some concretes are calculated and found to be in agreement with the previous experimental data.

In addition, some empirical and semi-empirical constitutive models in the previous literature, such as the series, parallel, the Hirsch and the Counto models are re-examined. It is found that each of these models can correspond well to a ubiquitiformal one under a certain complexity. Comparing with these empirical and semi-empirical constitutive models, the ubiquitiformal one can describe the constitutive behavior of bimaterials in a more all-round way.

\section{Acknowledgements}

This work was supported by the National Natural Science Foundation of China (11772056).

\section{References}

1. BAŽANT Z.P., YAVARI A., 2005, Is the cause of size effect on structural strength fractal or energetic-statistical? Engineering Fracture Mechanics, 72, 1-31 
2. Carpinteri A., Chinia B., Cornetti P., 2003, On the mechanics of quasi-brittle materials with a fractal microstructure, Engineering Fracture Mechanics, 70, 2321-2349

3. Carpinteri A., Cornetti P., 2002, A fractional calculus approach to the description of stress and strain localization in fractal media, Chaos, Solitons and Fractals, 13, 85-94

4. Counto U.J., 1964, The effect of the elastic modulus of the aggregate on the elastic modulus, creep and creep recovery of concrete, Magazine of Concrete Research, 16, 129-138

5. Davey K., Alonso Rasgado M.T., 2011, Analytical solutions for vibrating fractal composite rods and beams, Applied Mathematical Modelling, 35, 1194-1209

6. Hashin Z., 1962, The elastic moduli of heterogeneous materials, Journal of Applied Mechanics, 29, 1, 143-150

7. HÄFner S., Eckardt S., Luther T., 2006, Mesoscale modeling of concrete: Geometry and numerics, Computers and Structures, 84, 450-461

8. Hirsch T.J., 1962, Modulus of elasticity of concrete affected by elastic moduli of cement paste matrix and aggregate, Journal of the American Concrete Institute, 59, 427-452

9. Li C.Q., Zheng J.J., Zhou X.Z., MCCArthy M.J., 2003, A numerical method for the prediction of elastic modulus of concrete, Magazine of Concrete Research, 55, 6, 497-506

10. Li G.Y., Ou Z.C., Xie R., Duan Z.P., Huang F.L., 2016, A ubiquitiformal one-dimensional steady-state conduction model for a cellular material rod, International Journal of Thermophysics, $\mathbf{3 7}, 47,1-13$

11. Li J.Y., Ou Z.C., Tong Y., Duan Z.P., Huang F.L., 2017, A statistical model for ubiquitiformal crack extension in quasi-brittle materials, Acta Mechanica, 228, 2725-2732

12. Mandelbrot B.B., 1967, How long is the coast of Britain? Statistical self-similarity and fractional dimension, Science, 156, 636-638

13. Mandelbrot B.B., 1977, Fractals: Form, Chance, and Dimension, Freeman, San Francisco

14. Mandelbrot B.B., 1982, The Fractal Geometry of Nature, Freeman, New York

15. Mandelbrot B.B., Passoja D.E., Paullay A.J., 1984, Fractal character of fracture surfaces of metals, Nature, 308, 721-722

16. Ou Z.C., Li G.Y., DuAn Z.P., Huang F.L., 2014, Ubiquitiform in applied mechanics, Journal of Theoretical and Applied Mechanics, 52, 1, 37-46

17. Ou Z.C., Li G.Y., Duan Z.P., Huang F.L., 2019, A stereological ubiquitiformal softening model for concrete, Journal of Theoretical and Applied Mechanics, 57, 1, 27-35

18. Ou Z.C., Yang M., Li G.Y., Duan Z.P., Huang F.L., 2017, Ubiquitiformal fracture energy, Journal of Theoretical and Applied Mechanics, 55, 3, 1101-1108

19. Road Research Laboratory, 1950, Design of Concrete Mixes, 2nd ed. London, H.M. Stationery Office, pp. 16, Road Note No. 4

20. Stock A.F., Hannantt D.J., Williams R.I.T., 1979, The effect of aggregate concentration upon the strength and modulus of elasticity of concrete, Magazine of Concrete Research, 31, 109, $225-234$

21. Vilardell J., Aguado A., Agullo L., Gettu R., 1998, Estimation of the modulus of elasticity for dam concrete, Cement and Concrete Research, 28, 1, 93-101

22. Zheng J.J., Li C.Q., Zhou X.Z., 2006, An analytical method for prediction of the elastic modulus of concrete, Magazine of Concrete Research, 58, 10, 665-673 